\title{
La jolla, una estrategia campesina basada en el manejo del arrastre hídrico de sedimentos y la diversidad vegetal
}

\author{
The jolla, a peasant strategy based on the management of sediment water trawl and \\ plant diversity
}

Javier Gómez Díaz', Salvador Lozano Trejo ${ }^{1}$

\section{RESUMEN}

En este artículo se analizó el manejo de la jolla en la comunidad de San Pedro Quilitongo, Oaxaca. Se aplicaron entrevistas semiestructuradas considerando aspectos ambientales (forma de construcción, diversidad vegetal y manejo), sociales (composición de la unidad familiar, migración y mano de obra) y económicos (tendencia temporal, rendimientos y dinámica anual). Se observaron las tasas de pérdida-formación de suelo y se analizaron muestras de suelo en el laboratorio. La zona de jollas retiene el 51\% del suelo desprendido por acción del agua. El manejo consiste en la incorporación de estiércoles para aumentar la materia orgánica, la rotación de cultivos, el descanso de las áreas de cultivo y el uso del arado, generando un suelo propicio para el cultivo. Allí siembran maíz (cinco variedades), frijol, haba, calabaza, chícharo, chilacayota, y se identifican hasta seis combinaciones de cultivos. Los campesinos llevan a cabo la "mano vuelta" para complementar la mano de obra faltante producto de la migración.

Palabras clave: formación de suelo, tecnología tradicional, agricultura ecológica

\begin{abstract}
In this article the management of la jolla in the community of San Pedro Quilitongo, Oaxaca, was analyzed. Semi-structured interviews were applied considering environmental aspects (construction, plant diversity and management), social (family unit composition, migration and labor) and economic aspects (temporal trend, yields and annual dynamics) -soil formation, and soil samples were analyzed in the laboratory. The jollas area retains $51 \%$ of the soil released by water. The management consists in the incorporation of manures to increase organic matter, the rotation of crops, the rest of the cultivated areas and the use of the plow, generating a favorable soil for the crop. In which they plant maize (five varieties), beans, faba beans, squash, peas, chilacayota, identifying up to six crop combinations in them. The peasants carry out the "hand return" to complement the missing labor product of the migration.
\end{abstract}

Key words: soil formation, traditional technology, ecological agriculture

\section{Introducción}

En México, las nuevas tecnologías no se aplican por las condiciones de pobreza que existen, por lo cual la tecnología tradicional es la única posible. Puede deducirse entonces que la mejor opción en el sector mayoritario del agro es la recuperación, renovación, fortalecimiento y enriquecimiento de las tecnologías indígenas y campesinas, buscando reducir las desventajas de las tecnologías agrícolas tradicionales y rescatando las aportaciones del conocimiento de los indígenas y campesinos (Gómez et al., 1998).

Este tipo de tecnología, por sus antecedentes milenarios, aporta elementos para la implementación de una producción acorde con los ciclos naturales, una agricultura ecológica que contribuye a un desarrollo más sustentable. Algunos ejemplos de estas tecnologías campesinas son las relacionadas con el uso y manejo del agua (en forma de escorrentías) y suelo, denominadas tecnologías de riego no convencional (la jolla es una de ellas). En la región de la Mixteca hay una larga historia del manejo y uso del agua, y existen vestigios de las primeras técnicas de hace 2,800 años. Actualmente, pequeños grupos de población todavía tienen conocimientos hidrogeológicos e hidroagrícolas de inestimable valor y es crucial capitalizarlos para generar opciones de desarrollo a partir de ellos (Rivas et al., 2007). 
Palerm (2008) explica que la jolla es una técnica sencilla que consiste en la construcción de diques en la misma barranca. El mismo suelo que arrastra el agua de lluvia se va acumulando detrás del dique y posteriormente es utilizado para los cultivos (Figura 1). Se aprovecha, asimismo, la humedad residual que deja la misma corriente de agua.

Rivas et al. (2008) señalan que, al describir el comportamiento de una jolla, sobre todo en comparación con el funcionamiento productivo de un suelo de temporal, queda claro que solo en este tipo de espacios (las jollas) es posible la retención y almacenamiento de agua de lluvia. El tipo de suelo que generalmente predomina en una jolla es la arena que ayuda a infiltrar y conservar la humedad. Con las jollas se evita que con la erosión, el poco suelo existente se vaya de la comunidad. De acuerdo con Galindo (2008), la jolla es una técnica hidráulica para retener en los lechos de arroyos secos o barrancas el agua y suelo que arrastra consigo la escorrentía al registrarseuna lluvia torrencial.

Rivas et al. (2008) destacan que el sistema de jollas no se refiere a una pequeña superficie cultivada con maíz en el fondo de una barranca aislada. Se trata más bien de un sistema complejo de manejo de agua, suelos y especies vegetales que con el paso de los años puede generar superficies agrícolas de considerables dimensiones (Figura 2). El sistema de jollas modifica el paisaje natural para convertirlo en un paisaje antrópico de alto potencial productivo y estético que permite el arraigo en la región Mixteca.

Rivas et al. (2007) explican que las jollas se pueden hacer de tierra compactada con ayuda de maquinaria o de piedra acomodada en forma totalmente manual. El proceso consiste en levantar sobre el cauce de una cárcava o barranca, una pared o bordo, en seco. Se va acomodando la piedra o tierra de manera que se cierre la corriente del agua en el cauce de la cañada, para que cuando llueva, el mismo suelo que es arrastrado por la corriente del agua de lluvia se vaya deteniendo allí, formando un depósito. Un aspecto importante para la retención del suelo son los bordos que protegen a la jolla.

Los bordos agrícolas son hileras de plantas, tierra y/o piedras que separan campos agrícolas o áreas abiertas en los agroecosistemas. En México, los bordos se han utilizado desde la época prehispánica, principalmente como un método para prevenir la erosión. Los indígenas obtenían una gran variedad de productos de los magueyes cultivados en los bordos (Zuria y Gates, 2006). Por lo anterior, el objetivo de este artículo fue analizar el manejo de la jolla en la comunidad de San Pedro Quilitongo, Oaxaca.

\section{Materiales y métodos}

La Agencia de Policía de San Pedro Quilitongo (Figura 3) pertenece al municipio de Asunción
Nochixtlán, y se sitúa en la región de la Mixteca de Oaxaca, México. Se ubica en las coordenadas 97 $09^{\prime} 42^{\prime \prime}$ E y $17^{\circ} 27^{\prime} 52^{\prime \prime} \mathrm{N}$, a una altitud de $2.320 \mathrm{msnm}$ (INEGI, 2005). El clima corresponde al tipo C (WO") (W) big, que es un clima templado subhúmedo, y es el más seco de los templados con lluvias en verano. La precipitación pluvial varía de 300 a 750 mm con una distribución temporal errática entre junio y octubre, con periodos de sequía de 21 a 40 días entre julio y agosto.

La comunidad se encuentra en la región de la Mixteca, la cual ha sufrido un serio deterioro ambiental desde hace aproximadamente 500 años, que se ha visto acelerado durante las últimas cuatro décadas (Velásquez y León, 2006). Se estima que el $83 \%$ de los suelos de la Mixteca están ligera a moderadamente degradados y el 17\% muestran signos de erosión severa (Altieri et al., 2006). Desde el punto de vista ambiental, se encuentra en una región semiárida que se caracteriza por la deforestación y la erosión, donde predomina la producción campesina de autoabastecimiento con alto grado de marginación y altos índices de migración. Actualmente, la región Mixteca aporta el 26.28\% de la población migrante que sale de Oaxaca (IOAM, 2009).

La información se recabó a través de entrevistas semiestructuradas (Bonilla y Rodríguez (1997) a 13 familias campesinas que manejan la jolla en la comunidad de San Pedro Quilitongo. Las preguntas estaban orientadas a indagar los aspectos ambientales (forma de construcción, diversidad vegetal y manejo), sociales (composición de la unidad familiar, migración y mano de obra) y económicos (tendencia temporal, rendimientos y dinámica anual) de las familias que manejan la jolla.

En el aspecto ambiental, para determinar las tasas de pérdida-formación de suelo, se seleccionaron sitios donde se observaron los rasgos más visibles de erosión hídrica y las zonas donde se forman las jollas. En cada sitio se clavaron estacas graduadas de $80 \mathrm{~cm}$ de longitud, a diferentes distancias $(3,5,6,9 \mathrm{~m})$, siguiendo el cauce de los escurrimientos y cubriendo el área de jollas, con la finalidad de medir el nivel del suelo después de cada evento de lluvia.

La estimación de las tasas de pérdida-formación de suelo se realizó de acuerdo a los siguientes datos y fórmulas:

$$
\text { Volumen }\left(\mathrm{m}^{3}\right)=\text { Espesor }(\mathrm{m}) \text { Área }\left(\mathrm{m}^{2}\right)
$$

Espesor (m), el cual se obtuvo calculando el promedio del nivel de suelo perdido o ganado registrado en las estacas de cada sitio elegido.

Área $\left(\mathrm{m}^{2}\right)$, de cada sitio.

Densidad aparente (Ton $\mathrm{m}^{-3}$ ), determinado a partir de la densidad aparente $\left(\mathrm{g} \mathrm{cm}^{-3}\right)$ promedio de cada sitio.

Tasa de pérdida o ganancia $($ Ton $)=$ Volumen $\left(\mathrm{m}^{3}\right)$ Densidad aparente (Ton $\mathrm{m}^{-3}$ ) 


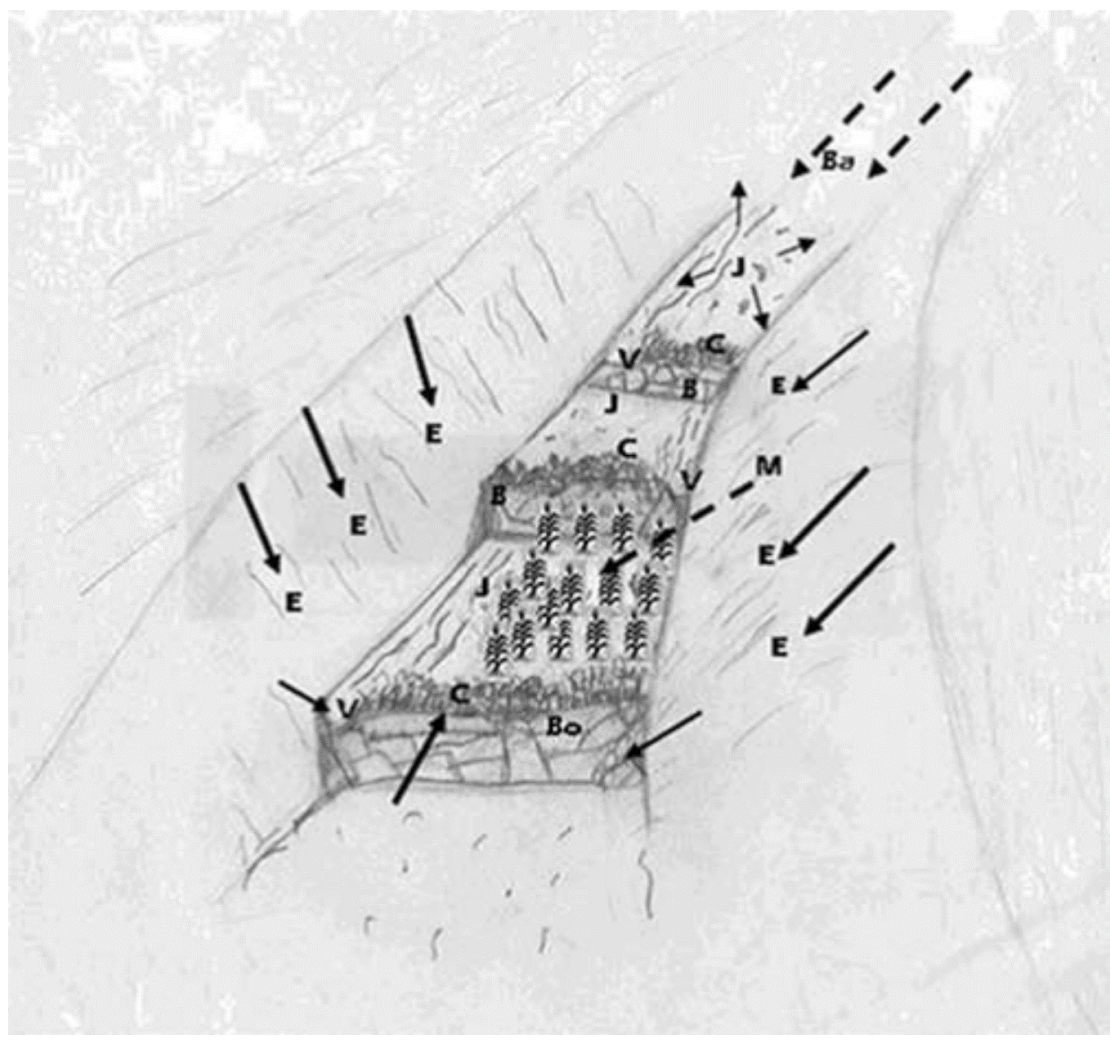

Figura 1. Conjunto de jollas en una barranca. Fuente: Rivas et al. (2008).

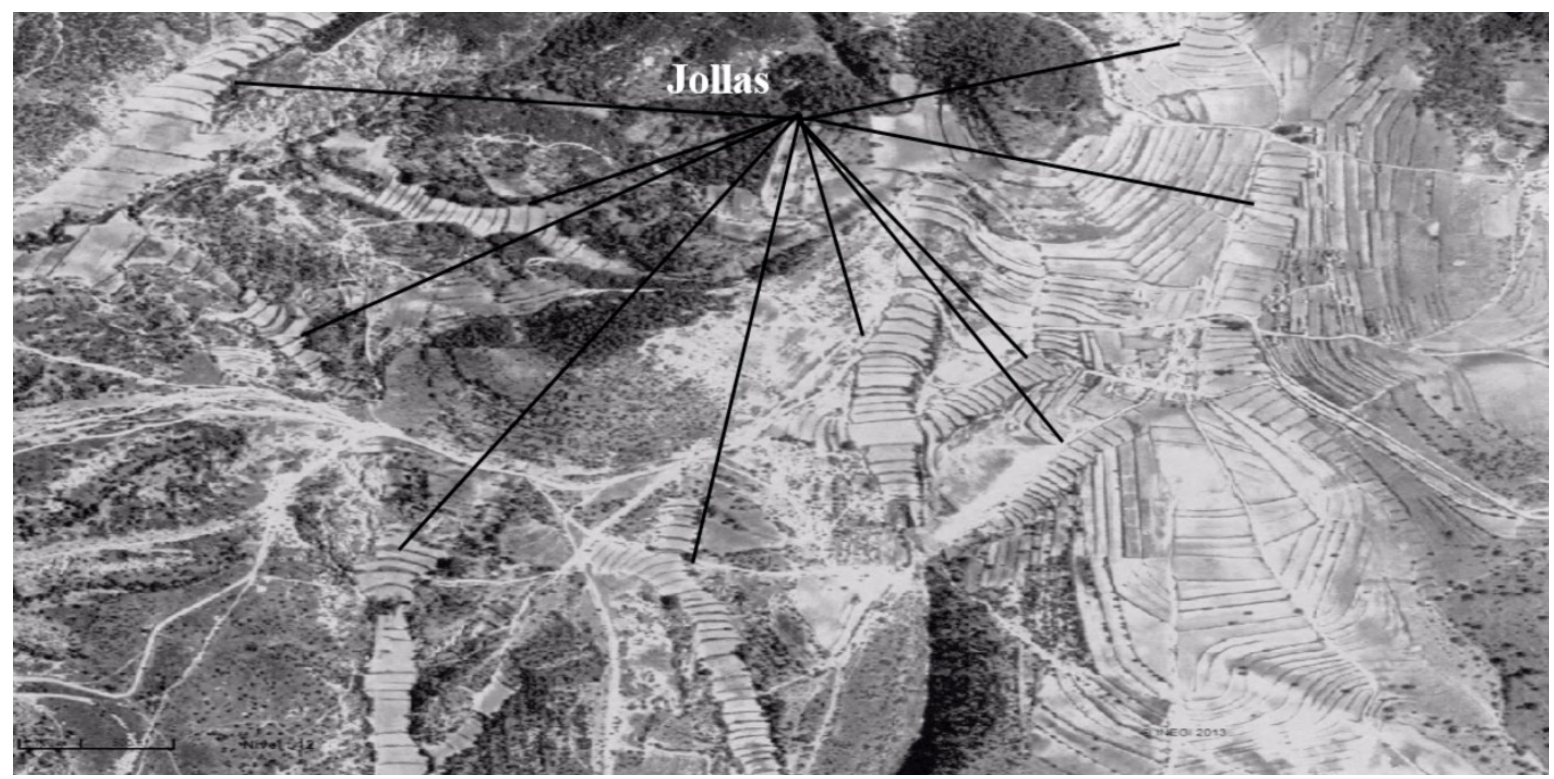

Figura 2. Jollas identificadas en la comunidad de estudio. Fuente: Elaboración propia 


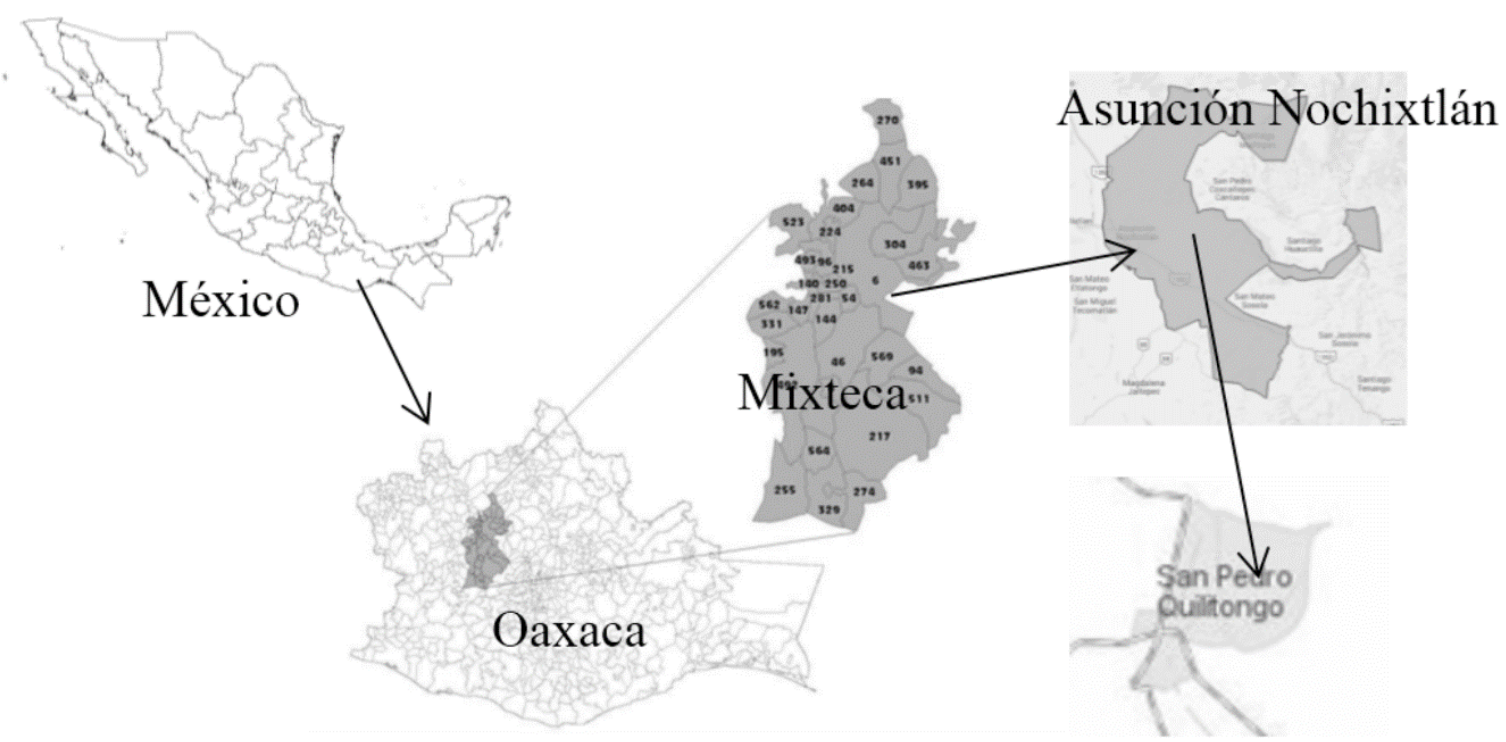

Figura 3. Ubicación de San Pedro Quilitongo. Fuente: Elaboración propia

Se tomó una muestra de suelo de cada uno de los sitios elegidos y se procedió al secado, tamizado y análisis (determinación de textura, densidad aparente y $\mathrm{pH}$ ). Esto se realizó en el Laboratorio de Diagnóstico Ambiental del Instituto Tecnológico del Valle de Oaxaca, de acuerdo con la Norma Oficial Mexicana NOM-021RECNAT-2000, que establece las especificaciones de fertilidad, salinidad y clasificación de suelos, estudios, muestreo y análisis.

\section{Resultados}

\section{Eje ambiental}

Para formar las jollas, en la comunidad de San Pedro Quilitongo, se aprovechan los sedimentos arrastrados de áreas aguas arriba, que son trasladados por erosión hídrica hasta las partes bajas. Allí, a través de la construcción de bordos de tierra (reforzados con la siembra de maguey, carrizo, etc.), se detiene el suelo que es arrastrado por la escorrentía, formando nuevos suelos de cultivo (con una profundidad mayor a un metro). Es necesario mencionar que se requiere un largo periodo de trabajo para que la jolla sea funcional. Una vez que sea productiva es necesario darle mantenimiento constantemente, principalmente en la zona de los bordos, ya que si no se hace puede romperse y el suelo acumulado será arrastrado por la escorrentía, alterando su estructura y funcionamiento.

De acuerdo al análisis realizado sobre la pérdidaformación de suelo, la zona de jollas retiene el $51 \%$ del suelo desprendido por acción del agua. Como se observa en la Tabla 1, la tasa de erosión es de 194.553 t ha-1, y de esta cantidad de suelo que se desprende, las jollas captan 100.444 tha-1 de suelo.

El suelo que forma a la jolla tiene cierto grado de artificialidad, ya que sin los bordos sería arrastrado por la escorrentía. Por ello, un aspecto importante es el manejo del suelo que realizan los campesinos con el fin de hacerlo funcional para el cultivo. Este manejo consiste en la incorporación de estiércoles para aumentar la materia orgánica, la rotación de cultivos, el descanso de las áreas de cultivo y el uso del arado. Con ello generan un suelo propicio para el cultivo, con un $\mathrm{pH}$ promedio de 7.8, el cual se encuentra en el rango óptimo para la mayoría de los cultivos. La densidad aparente (Tabla 1) refleja suelos poco compactados, la textura tiene la característica de retener humedad. La cantidad de materia orgánica promedio se considera deficiente (3\%), por lo cual, los campesinos incorporan estiércoles y desechos orgánicos (provenientes de las cocinas), con lo que tratan de dar solución a la baja cantidad de materia orgánica presente en el suelo. En los últimos años se han incorporado abonos verdes (principalmente las malezas que nacen en el descanso de la parcela) para complementar los nutrientes del suelo.

La diversidad es el sustento de los cultivos que se realizan en la jolla, y se observa principalmente la milpa como una estrategia de producción múltiple con la cual buscan obtener un número mayor de productos a través de la combinación de especies. La milpa permite la obtención de diferentes productos que conforman 
Tabla 1. Tasas de pérdida-formación en cada sitio muestreado.

\begin{tabular}{ccccccccc}
\hline \multirow{2}{*}{ Sitio } & \%Arena & \%Arcilla & \%Limo & Textura & D A & pH & formación (+) (Ton) \\
\hline Cárcava & 50.68 & 20.76 & 28.56 & Franca & 1.332 & 8.06 & -191.408 \\
Jolla & 50.68 & 20.76 & 28.56 & Franca & 1.332 & 8.06 & 57.842 \\
Cárcava & 50.3 & 22.15 & 27.55 & $\begin{array}{c}\text { Franco arcillo } \\
\text { arenosa }\end{array}$ & 1.209 & 7.77 & -3.145 \\
Jolla & 50.3 & 22.15 & 27.55 & $\begin{array}{c}\text { Franco arcillo } \\
\text { arenosa }\end{array}$ & 1.209 & 7.77 & 14.624 \\
Jolla & 43.70 & 27.39 & 28.92 & Franco arcillosa & 1.135 & 7.85 & 27.978 \\
\hline
\end{tabular}

Fuente: Elaboración propia.

la dieta de los campesinos, incluso de especies no sembradas como los quelites, verdolagas y la captura de chapulines. Este sistema proporciona maíz, frijol, haba, calabaza, chícharo, chilacayota y forraje. Lo anterior producto de diversas combinaciones que realizan en la milpa: maíz, frijol, haba y calabaza; frijol y maíz; maíz, frijol y calabaza; maíz, frijol y haba; maíz y calabaza; maíz, frijol y chícharo.

Esta diversidad también se aprecia en los bordos de las jollas, donde se siembra guajes, nopales, carrizo y maguey, con un doble propósito: primero, para fortalecer los bordos de las jollas, y segundo, para obtener distintos productos. De estos el más importante es el pulque, debido a que los campesinos se dedican también a la venta de esta especie, con la cual complementan las necesidades de sus familias. Mediante la diversidad vegetal, los campesinos buscan obtener variados productos dentro de los ciclos productivos, aun cuando existan perturbaciones ambientales.

En el caso del maíz los campesinos utilizan diversas variedades (blanco, azul, pinto, amarillo y cajete), para afrontar las perturbaciones ambientales como son lluvias erráticas y en ocasiones largos periodos de sequía, con el fin de asegurar la producción. A pesar de que la jolla en un principio era construida para la siembra de maíz cajete (que es de ciclo largo), los campesinos optaron por sembrar variedades de ciclo más corto para asegurar sus cosechas.

Debido a los problemas de degradación de los recursos que sufre la comunidad, se han emprendido acciones para revertir esta situación, como son la reforestación, la construcción de zanjas trincheras y jagüeyes. Los campesinos tienen la visión de que todas estas acciones no solo benefician a la comunidad, sino que impactan a todos los seres vivos que habitan en el planeta. Otro aspecto a resaltar es el uso mínimo de envases de plástico, por el impacto en el ambiente que tienen, al igual que la utilización de fertilizantes químicos, que está restringida en la comunidad porque, de acuerdo a su percepción, "matan la tierra". La reforestación se realiza con plantas de pino, debido a que esta es la especie que proporciona la Comisión Nacional
Forestal (CONAFOR). Los habitantes preferirían reforestar con especies propias de la región.

En el aspecto productivo, se han adoptado innovaciones eco-tecnológicas como la incorporación de abonos verdes, además de nuevos criterios para la selección de semillas (plantas que produzcan dos mazorcas), abonado mateado con el que se permite abonar más superficie de cultivo, la captación de agua de lluvia para dar respuesta a la falta de agua y la construcción de estufas ahorradoras de leña, lo que disminuye la presión sobre las zonas forestales.

\section{Eje económico}

En el área económica, los campesinos que manejan la jolla en San Pedro Quilitongo se dedican al cultivo de maíz, frijol y trigo de temporal para el autoconsumo. Estos cultivos a través del tiempo han disminuido su rendimiento. Por ejemplo, en 1940 se cosechaba de 1-2 toneladas de trigo, y actualmente los rendimientos son de $450 \mathrm{~kg} \mathrm{ha}^{-1}$ en promedio. Algo similar sucede con los rendimientos del maíz y frijol.

El principal producto por el cual obtienen recursos es el pulque. Lo venden los días domingos en la plaza de Asunción Nochixtlan, por litro y por jícara. Es en esta plaza donde obtienen mayores ingresos, ya que a la comunidad llegan a comprar el pulque los mayoristas, pagándolo a muy bajo precio. En la comunidad el precio del litro es de 5 pesos, mientras que en la plaza venden el litro a 20 pesos.

El rendimiento del pulque es 301 planta $^{-1}$ en promedio. Sin embargo, el periodo de producción de la planta en los últimos ha disminuido, principalmente por la falta de lluvias, pero también por problemas de plaga de una especie de ardilla y ratas que se comen las raíces de los magueyes.

La dinámica económica a través del año estableció que los meses de mayor ingreso son de septiembre a diciembre. Los principales ingresos son por el jornaleo (área de la construcción) y por la venta de pulque. Otra fuente de ingresos son los apoyos gubernamentales, así como la venta de animales (en menor proporción). 
Los mayores egresos se dan en los meses de marzoabril (cuando se contrata el tractor para las labores productivas). La falta de dinero en estos meses provoca que se deje de sembrar el $25 \%$ aproximadamente de la superficie para cultivar en el ciclo en curso. Otros dos egresos importantes para la familia campesina se realizan en el ingreso a la escuela y en las festividades de la comunidad.

Al depender del tractor para las labores, los campesinos se ven afectados por los altos precios del combustible, aspecto que ellos no pueden controlar, pero tampoco tienen la alternativa de retornar al uso de la yunta, ya que existe un déficit de forraje. Las familias campesinas que poseen yunta compran forraje para los animales, lo que representa una inversión económica. Lo anterior influye en el número de animales que tienen las unidades familiares, lo que a su vez afecta la disponibilidad de estiércol para los cultivos.

\section{Eje social}

La migración es un fenómeno que impacta a la comunidad de San Pedro Quilitongo, y por consiguiente a las familias que manejan la jolla. Las personas migran hacia la ciudad de Oaxaca de Juárez o a la ciudad de México, en busca de mejores oportunidades para aumentar sus ingresos. Los migrantes trabajan como obreros (albañiles y plomeros principalmente), como comerciantes (establecidos y no establecidos) y como prestadores de servicios (profesores, meseros, etc.). La migración reduce el tamaño de la familia en la comunidad, la cual está compuesta por tres integrantes (padre, madre e hijo).

La fuerza productiva y reproductiva de la comunidad se encuentra fuera de ella, lo que se refleja en la permanencia de los productores mayores de 40 años. Debido a la migración el tamaño de la unidad familiar se ve reducido, y por esto los campesinos establecen otras estrategias para completar el déficit de mano de obra a través de "la mano vuelta".

Todas las familias mantienen este código de ayuda, con el cual pueden complementar parte de la mano de obra que hace falta por la migración. Mediante "la mano vuelta" las familias campesinas se organizan con base en los requerimientos de la producción para el trabajo y en equipo, con un alto grado de solidaridad.

Sibien "lamanovuelta" contribuyeacomplementar la mano de obra que hace falta por la migración, un aspecto que no puede ser reemplazado es la transferencia de conocimientos. Como ya se mencionó, la fuerza productiva se encuentra fuera de la comunidad y por consiguiente la transferencia de conocimientos en las unidades de producción es afectada porque los hijos están dejando de participar en las actividades productivas y culturales. Con esto se ponen en riesgo los conocimientos acumulados a través del tiempo.

\section{Conclusiones}

La jolla contribuye a retener el $50 \%$ de los suelos arrastrados por acción hídrica, gracias al manejo que realizan los campesinos de las corrientes hídricas en zonas de desprendimiento de suelo, con lo que también crean superficies agrícolas aptas para sus cultivos.

La diversidad vegetal cultivada y las distintas combinaciones de cultivos que se realizan en la jolla, así como el manejo del suelo, permiten obtener cosechas. Sin embargo, en los últimos años, los rendimientos se han visto afectados por la disminución de lluvias en la región.

La falta de opciones para obtener recursos, que complementen las cosechas así como la venta de pulque, impulsa la migración de las familias que manejan la jolla, la cual se ve afectada porque se rompe la transmisión de los conocimientos hidro-agrícolas, ya que la fuerza productiva se encuentra fuera de la comunidad.

\section{Literatura citada}

Altieri, M., Fonseca, A. S.; Caballero, J. J.; Hernández, J. J.

2006. Manejo del agua y restauración productiva en la región indígena mixteca de Puebla y Oaxaca. Editorial Banco Mundial. México D.F. 100 p.

Bonilla, C. E.; Rodríguez, S. P.

1997. Más allá del dilema de los métodos. La investigación en las ciencias sociales. Editorial Norma. Buenos Aires, Argentina. 411 p.

Galindo, E. E.

2008. Captación de agua de lluvia y retención de suelo en jollas en el parte aguas de las cuencas de los ríos Actopan y Amajac. Boletín del archivo histórico del agua, 13: 17-20.

Gómez, G. G.; Ruiz, G. J.; Bravo, G. S.

1998. Tecnología tradicional indígena y la conservación de los recursos naturales. En: Ordóñez C. J. E. (Coor.). Balance y perspectiva del derecho socialy los pueblos indios de Mesoamérica. Ed. Universidad Nacional Autónoma de México, D.F., México. 142 p.

INEGI.

2005. Anuario estadístico y geográfico por entidad federativa 2005. Ed. Instituto Nacional de Estadística, Geografía e Informática. D.F., México. 570 p. IOAM

2009. Estadísticas de la población migrante oaxaqueña 2009. Ed. Instituto Oaxaqueño de Atención al Migrante. Oaxaca, México. $218 \mathrm{p}$.

OEIDRUS.

2005. Tarjeta distrital de información estadística básica. Ed. Oficina Estatal de Información para el Desarrollo Rural Sustentable. Oaxaca, México. 56 p. 


\section{Palerm, V. J.}

2008. Jollas. Boletín del archivo histórico del agua, 13:3-6.

Rivas, G. M.; Palerm, V. J.; Vázquez, C. G.; Cuevas, S. J.

2007. Las jollas, sistema agrícola y tecnología ancestral en la mixteca oaxaqueña. VI congreso mexicano de etnobiología. Oaxaca México. 6 p.

Rivas, G. M.; Rodríguez, H. B.; Palerm, V. J.

2008. El sistema de jollas una técnica de riego no convencional en la mixteca. Boletín del archivo histórico del agua, 13: 6-16.
Velásquez, C. J.; León, S. J.

2006. Centro de Desarrollo Integral Campesino de la Mixteca CEDICAM: una organización de campesinos para campesinos. LEISA, 22 (2): 24-26.

Zuria, I.; Gates, J. E.

2006. Vegetated field margins in Mexico: Their history, structure and function, and management. Human Ecology. 34: 53-77. 
